\title{
Cryopreservation of Gonadal Germ Cells (GGCs) from the Domestic Chicken Using Vitrification
}

\author{
Yusaku Kohara, Yukio Kanai and Atsushi Tajima \\ Graduate School of Life and Environmental Sciences, University of Tsukuba, \\ 1-1-1 Ten-noh Dai, Tsukuba, Ibaraki 305-8572, Japan
}

\begin{abstract}
We cryopreserved gonadal germ cells (GGCs) collected from 7-day-old chick embryos using the vitrification method. Gonadal germ cells were suspended in vitrification medium and plunged directly into liquid nitrogen. The recovery rates of GGCs after freezing via vitrification and slow-freezing methods were $36.8 \pm 1.5$ and $56.7 \pm 3.0 \%$, respectively. The survival rates of GGCs in the unfrozen control, vitrified and slow-frozen cells were $98.0 \pm 0.2,85.8 \pm 1.2$ and $91.2 \pm 2.8 \%$, respectively. The recovery and survival rates were significantly lower for vitrified GGCs than for slow-frozen GGCs $(p<0.05)$. To evaluate the migratory ability of cryopreserved GGCs, frozen/thawed GGCs were labeled with PKH2 fluorescent dye, and 20 GGCs were transferred into 2-day-old chick embryos. After incubating for three days, the embryonic gonads were collected and the number of PKH2-labeled GGCs per chick was counted. These values for the control, vitrified and slow-frozen cells were $96.5 \pm 23.9,102.0 \pm 39.0$ and $90.0 \pm 34.2$ cells, respectively. No significant differences were observed among treatments $(p>0.05)$. These results demonstrate that it is possible to cryopreserve GGCs using vitrification, although the recovery and survival rates are lower than those observed using the slow-freezing method.
\end{abstract}

Key words: chicken, cryopreservation, gonadal germ cells, migratory ability, vitrification

J. Poult. Sci., 45: 57-61, 2008

\section{Introduction}

As the number of endangered avian species increases in both wild (IUCN, 2006) and domesticated populations (Hodges, 2006), it is increasingly important to develop methods to conserve avian genetic resources. In addition, avian genetic resources developed for various research purposes need to be conserved for our future use. Furthermore, the genetic material of commercial parent stocks needs to be preserved to reduce the risk of losses as a result of unexpected disease outbreaks and accidents.

In avian species, the cryopreservation of semen has already been achieved in the chicken (Polge, 1951), turkey (Bakst and Sexton, 1979), duck (Maeda et al., 1984) and some non-domesticated avian species such as the Sandhill Crane (Gee et al., 1985). In contrast, an effective method for the cryopreservation of avian oocytes and embryos has not yet been developed. Because of the unique physiological and anatomical characteristics of the ovum, a method other than freezing is required for the preservation of oocytes.

Primordial germ cells (PGCs) are undifferentiated germ cells that subsequently differentiate into spermatogonia in

Received: April 26, 2007, Accepted: August 10, 2007

Correspondence: A. Tajima, Graduate School of Life and Environmental Sciences, University of Tsukuba, Tsukuba, Ibaraki 305-8572,

Japan. (E-mail: tajima@sakura.cc.tsukuba.ac.jp) the testis or into oogonia in the ovary. PGCs originate from the epiblast (Eyal-Giladi et al., 1981) and appear in the area pellucida (Swift, 1914). After the development of the vascular system, PGCs circulate along with embryonic blood cells and finally migrate into the embryonic gonads (Ukeshima and Fujimoto, 1984; Kuwana, 1993; Kuwana and Rogulska, 1999). After migration, PGCs are called gonadal germ cells (GGCs). Tajima et al. (1993) produced germline chimeras by transferring PGCs from White Leghorn into Rhode Island Red chickens, and vice versa. Naito et al. (1994) produced germline chimeras by transplanting cryopreserved PGCs. Tajima et al. (1998) also produced germline chimeras using cryopreserved GGCs collected from 5-day-old embryos. All of these studies used the slow-freezing method and the Bicell biofreezing vessel to cryopreserve germ cells. To optimize the freezing rate in this method, the Bicell container needs to be placed in a deep-freeze maintained at $-80^{\circ} \mathrm{C}$.

Rall and Fahy (1985) demonstrated the cryopreservation of mouse embryos via vitrification. Vitrification is an ultra-rapid cryopreservation technique that uses high concentrations of cryoprotectants that inhibit the formation of ice crystals. Nakao et al. (1997) developed a simplified vitrification method that uses a modified freezing solution; this method successfully produced young mice from vitrified embryos. However, the vitrification of chick germ cells has not been reported until now. Therefore, we attempted to cryopreserve avian GGCs using the vitrific- 
ation method.

\section{Materials and Methods}

\section{Experiment 1: Vitrification of GGCS}

All equipment that came into direct contact with germ cells was coated with silicone (Sigma Coat SL-2; Sigma, St. Louis, MO, USA). Fertilized Y3-strain White Leghorn (WL) eggs were incubated at $37.8^{\circ} \mathrm{C}$ for 7 days. This strain was obtained from the Takikawa Animal Experiment Station (Takikawa, Hokkaido, Japan) and maintained at the Agricultural and Forestry Research Center at the University of Tsukuba, Japan. After incubation, the embryos were dissected off the egg yolks and washed with phosphate-buffered saline that did not contain $\mathrm{Mg}^{2+}$ and $\mathrm{Ca}^{2+}$ (PBS(-), 05913; Nissui Pharmaceutical Co., Tokyo, Japan). The head and tail of each embryo were fixed with needles, and the left embryonic gonad was collected under an Olympus dissecting microscope (SZH; Tokyo, Japan). We used five embryos.

The collected gonads were sliced using the tip of a $30 \mathrm{G}$ dental needle (DN-3021K; Terumo, Tokyo, Japan) in 30 $\mu l$ of PBS (-). The sliced gonads were placed into $1.5-\mathrm{m} l$ centrifuge tubes containing $0.05 \%$ trypsin (203-11302; Wako, Osaka, Japan). The tubes were incubated at $37.8^{\circ} \mathrm{C}$ for 20 minutes.

After the trypsin treatment, an equal volume of bovine serum (B-0771; Sigma, St. Louis, MO, USA) was added to the centrifuge tubes and agitated to form a cell suspension containing GGCs. To remove the large cell clusters and undigested tissue fragments, the cell suspension was filtered through a $20-\mu \mathrm{m}$ nylon mesh filter (CMN-20-D; Small Parts Inc, Florida, USA) and centrifuged at $200 g$ for 10 minutes.

After centrifugation, the supernatant was removed, and an equal volume of minimum essential medium (MEM, M0769; GIBCO BRL, New York, USA) containing 10\% bovine serum was added. The cell suspension was allocated into the one of the three experimental treatments: vitrification, slow-freezing, or unfrozen control.

\section{a) Vitrification method}

Vitrification was performed according to the method described by Nakao et al. (1997), with some modifications. The germ cell suspension was centrifuged at $500 \mathrm{~g}$ at $4^{\circ} \mathrm{C}$ for 5 minutes, and the supernatant was replaced with $20 \mu l$ of PB1 medium (Nakao et al., 1997) containing 10\% DMSO. This step was repeated twice. The sample was then incubated in ice water for 5 minutes, after which $10 \mu \mathrm{l}$ of the cell suspension was placed into a sterile cryogenic vial (5000-0012; Nalgene Co., NY, USA), followed by the slow addition of $90 \mu l$ of DAP213 medium (Nakao et al., 1997). The vial was incubated in ice water for 5 minutes and then plunged into liquid nitrogen $\left(-196^{\circ} \mathrm{C}\right)$. After storing the sample for $1-3$ weeks, the sample was thawed by adding $500 \mu \mathrm{l}$ of PB1 medium containing $0.25 \mathrm{M}$ sucrose and warmed to $37^{\circ} \mathrm{C}$.

The frozen sample was then incubated in a water bath maintained at $37^{\circ} \mathrm{C}$, after which it was transferred to a 1.5 $\mathrm{m} l$ centrifuge tube and centrifuged at $800 \mathrm{~g}$ for 5 minutes at $37.8^{\circ} \mathrm{C}$. The supernatant was removed, and $50 \mu l$ of $\mathrm{PB}$ 1 medium containing $0.25 \mathrm{M}$ sucrose was added.

\section{b) Slow-freezing Method}

Slow-freezing was conducted according to the method described by Tajima et al. (1998), with some modifications. The sample was centrifuged at $500 \mathrm{~g}$ at $4{ }^{\circ} \mathrm{C}$ for 5 minutes. The supernatant was removed, and $20 \mu \mathrm{l}$ of MEM supplemented 10\% DMSO (MEM-D) was added. This step was repeated twice.

The cell suspension was incubated in ice water for 5 minutes, after which $20 \mu \mathrm{l}$ of the cell suspension was placed into a sterile cryogenic vial. The vial was placed into a biofreezing vessel (Bicell; Nihon Freezer Co., Tokyo, Japan) and then into a deep-freezer (ULT 1785-5D12; Revco, Inc., NC, USA) at $-80^{\circ} \mathrm{C}$ for at least $2 \mathrm{~h}$. After freezing, the cryogenic vial was removed from the freezing vessel, placed in liquid nitrogen $\left(-196^{\circ} \mathrm{C}\right)$ and stored for 1-3 weeks. The sample was thawed at room temperature and diluted stepwise by adding 20, 40 and 80 $\mu l$ of MEM. The diluted samples were pipetted into 1.5 $\mathrm{m} l$ centrifuge tubes and centrifuged at $800 \mathrm{~g}$ at $37.8^{\circ} \mathrm{C}$ for 5 minutes. After centrifugation, the supernatant was replaced with $200 \mu l$ of MEM.

\section{c) Control}

In the control, the samples were centrifuged at $800 \mathrm{~g}$ at $4^{\circ} \mathrm{C}$ for 5 minutes, after which the supernatant was replaced with $20 \mu l$ of MEM. This step was repeated twice. A $10 \mu l$ aliquot of sample was then diluted in $90 \mu l$ of MEM.

\section{Observation}

The viability of GGCs was evaluated three times for each sample using trypan blue staining. The recovery rate (the number of GGCs after freezing divided by number of GGCs before freezing) was calculated in triplicate for each sample. Gonadal germ cells were identified by the same morphological criteria as circulating PGCs, i.e., large granulated cells with large nuclei (Minematsu et al., 2004).

Statistical Analysies

The data were analyzed using one-way analysis of variance (ANOVA) followed by Duncan's multiple range tests.

\section{Experiment 2: The Migratory ability of vitrified GGCs}

Approximately 40 frozen/thawed GGCs were collected using a fine glass pipette and incubated in medium containing PKH2 fluorescent dye (ZPKH2-GL; Zynaxis, PA, USA) at $37.8^{\circ} \mathrm{C}$ for 5 minutes. After staining, 20 GGCs were collected and injected through a window into the dorsal aorta of stage 13-14 embryos (Hamburger and Hamilton, 1951). Eggshell windows were prepared 1-2 hours prior to germ cell injection; during this time, approximately $2.5-4 \mu \mathrm{l}$ of blood was removed from the embryo using a fine glass pipette.

After germ cell injection, the eggshell window was sealed with plastic tape, and the embryo was allowed to develop in an incubator for three days. The collection of 
GGCs from embryonic gonads was conducted according to the method described by Minematsu et al. (2004), with some modifications. After incubation, the gonads were collected from the GGC recipient embryos under a dis-

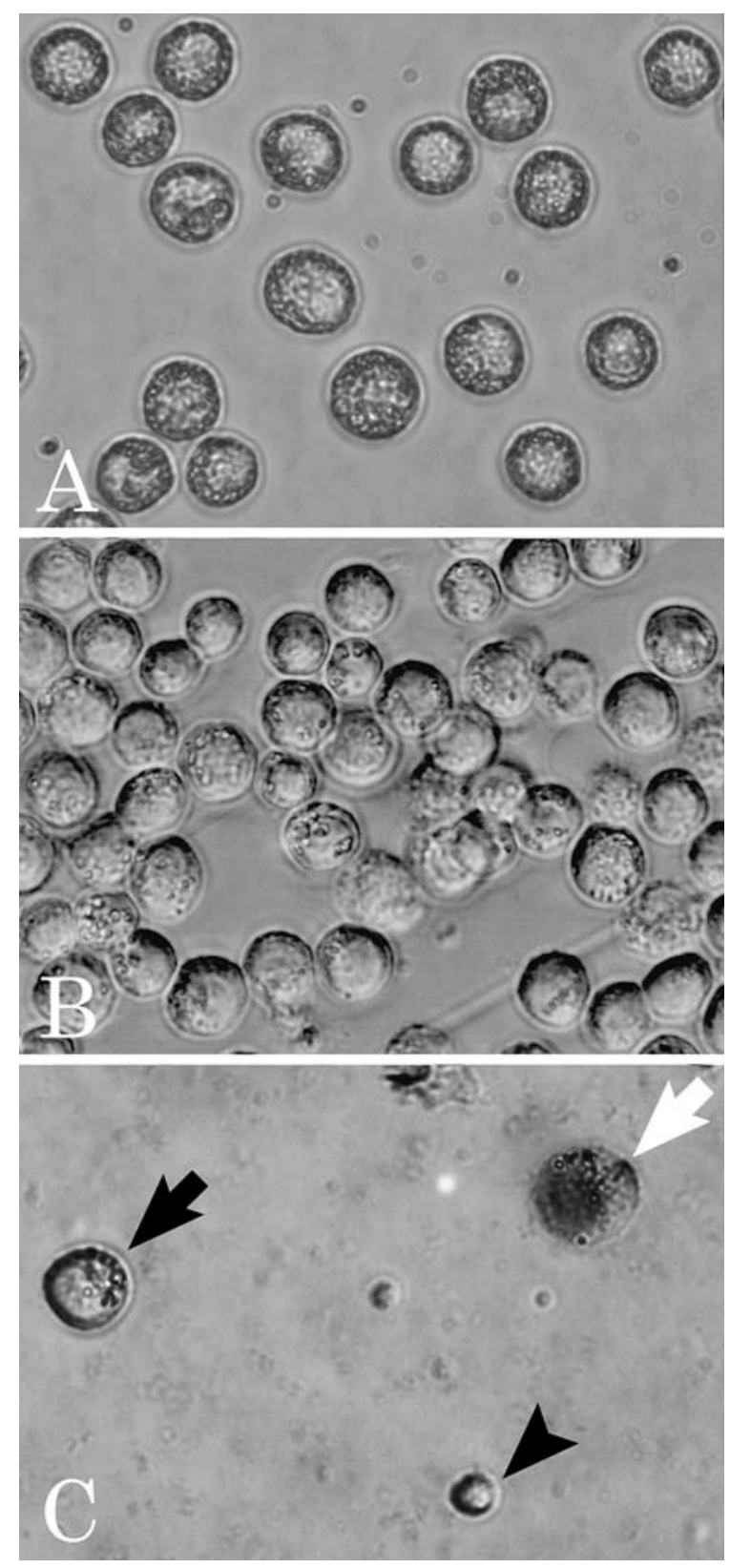

Fig. 1. Morphological characteristics of intact GGCs and trypan blue stained GGCs after cryopreservation. A: PGCs collected from dorsal aorta of 2-day-old embryos. B: GGCs collected from gonad of 7-day-old embryo. C: Frozen-thawed GGCs with Trypan blue staining. Black arrow: trypan blue unstained live GGC: White arrow: trypan blue stained dead GGC. Black arrowhead: somatic cell.

PGCs: Primordial Germ Cells, GGCs: Gonadal Germ Cells. secting microscope and placed into $30 \mu \mathrm{l}$ of PBS (-). After slicing the gonads, an equal volume of $0.05 \%$ trypsin was added into the PBS $(-)$ containing gonadal suspension, which was then incubated at $37.8^{\circ} \mathrm{C}$ for 10 minutes.

The suspension was diluted and mixed with an equal volume of bovine serum. The suspension was placed on the HTCS and observed under a fluorescence microscope (IMT2RFC; Olympus, Tokyo, Japan) using a 490-nm excitation filter to count the number of fluorescent GGCs.

The migratory ability of frozen/thawed GGCs was determined by counting the total number of fluorescent GGCs in the recipient gonads. The data were analyzed using Chi-square $\left(\chi^{2}\right)$ tests. The proliferation rate of GGCs was estimated by calculating the ratio of the number of fluorescent GGCs to the number of transferred GGCs.

\section{Results}

\section{Experiment 1}

As shown in Figure 1, the morphology of GGCs was similar to circulating PGCs. The survival of cryopreserved GGCs was determined using trypan blue staining (Fig. 1) and the survival rates of GGCs in the control, vitrified and slow frozen cells were 98.0 $\pm 0.2,85.8 \pm 1.2$ and $91.2 \pm 2.8 \%$, respectively (Table 1 ); there was no significant difference among treatments $(p<0.05)$. The recovery rates of vitrified and slow-frozen GGCs were 36.8 \pm 1.5 and $56.7 \pm 3.0 \%$, respectively. The recovery rate of vitrified GGCs was significantly lower than that of the slow-frozen cells $(p<0.05)$.

\section{Experiment 2}

For the migratory ability of transferred GGCs (Table 2 ), the proportion of recipient embryos with fluorescent GGCs from the control, vitrified and slow-frozen samples were $80.0,80.0$ and $60.0 \%$, respectively.

The number of fluorescent GGCs per embryo from the control, vitrified and slow-frozen samples were 96.5士 $23.9,102.0 \pm 39.0$ and $90.0 \pm 34.2$ cells, respectively $(p>$ $0.05)$. No significant difference in proliferation rate was observed among the treatment groups; the values for the control, vitrified and slow-frozen GGCs were 4.8, 5.1 and 4.5 times, respectively.

Table 1. Survival and recovery rates for GGCs after cryopreservation

\begin{tabular}{lccc}
\hline \hline & Survival rate $(\%)$ & & Recovery rate $(\%)$ \\
\cline { 2 - 2 } Control & $98.0 \pm 0.2^{\mathrm{a}}$ & & - \\
Vitrification & $85.8 \pm 1.2^{\mathrm{c}}$ & & $36.8 \pm 1.5^{\mathrm{b}}$ \\
Slow-freezing & $91.2 \pm 2.8^{\mathrm{b}}$ & & $56.7 \pm 3.0^{\mathrm{a}}$ \\
\hline
\end{tabular}

Recovery rate; (the rate of frozen GGCs/unfrozen GGCs) $\times 100$.

${ }^{a-c}$ : Values with different letters within columns are significantly different $(P<0.05)$. GGCs: Gonadal germ cells. 
Table 2. Migratory ability of transferred GGCs

\begin{tabular}{lccc}
\hline \hline & $\begin{array}{c}\text { Recipient embryo with } \\
\text { fluorescent GGCs }\end{array}$ & $\begin{array}{c}\text { Number of fluorescent } \\
\text { GGCs per embryo }\end{array}$ & $\begin{array}{c}\text { Proliferation rate } \\
(\%)^{1}\end{array}$ \\
\hline Control & $80 \%(4 / 5)$ & $96.5 \pm 23.9$ & 4.8 \\
Vitrification & $80 \%(4 / 5)$ & $102.0 \pm 39.0$ & 5.1 \\
Slow- freezing & $60 \%(3 / 5)$ & $90.0 \pm 34.2$ & 4.5 \\
\hline
\end{tabular}

Proliferation rate; (the ratio of the number of fluorescent GGCs to the number of transferred GGCs) $\times 100$. GGCs: Gonadal germ cells.

${ }^{1)}$ No significant difference among groups was detected using a $\chi^{2}$ test $(P>0.05)$.

\section{Discussion}

Even though the survival rate of vitrified GGCs was significantly lower than that of slow-frozen cells $(p<$ 0.05 ), it was still possible to retrieve viable GGCs that could successfully migrate to the embryonic gonad after they were transferred into the blood stream of 2-day-old embryos.

We encountered a significant disadvantage of vitrification: the recovery rate of vitrified cells was significantly lower than that of slow-frozen cells. However, because the method that we used was optimized for the vitrification of mouse embryos, it may be possible to improve the recovery rate of avian germ cells through a similar optimization process.

Several cryoprotectants have been used in freezing mammalian germ cells, e.g., ethylene glycol (EG) and polyvinyl-pyrrolidone for mouse oocytes and embryos (Leibo and Oda, 1993). EG, Ficoll and sucrose have been used to cryopreserve mouse embryos (Kasai et al., 1990) and bovine embryos (Tachikawa et al., 1993). A combination of EG and DMSO has been used to cryopreserve bovine oocytes (Vajta et al., 1998). DMSO has also been used in combination with acetamide and propylene glycol to cryopreserve mouse embryos (Nakao et al., 1997). An extensive study into the selection and concentrations of cryoprotectants is required to optimize the vitrification methods for avian germ cells.

The superficial dimensions of the freezing vessel strongly affect the cooling rate, making this an important factor in the optimization of vitrification. Recent studies have investigated the effects of several freezing vessels in working with mouse oocytes and embryos, including cryotubes (Nakao et al., 1997), glass micropipettes (Kong et al., 2000), the open pulled straw (Kong et al., 2000) and the closed pulled straw (Chen et al., 2001). The low recovery rates that we observed after vitrification could be improved by experimenting with different freezing vessels.

Shaw et al. (1992) studied the effect of exposure time to cryoprotective agents prior to vitrification on post-thaw survival and developmental ability in mouse oocytes. They found that shorter exposure times before vitrification corresponded to increased survival rates and developmental ability.

In Experiment 2, PKH2-positive GGCs, which we as- sumed were the injected GGCs, were observed in all treatments. No significant differences in migratory ability were observed among treatments. In addition, the number of fluorescent GGCs in all treatments was approximately 90-100 cells, which is $4.5-5$ times the number of injected cells. These results suggest that vitrified GGCs have normal migratory ability, as well as the ability to proliferate in the embryonic gonads.

In conclusion, vitrification is a simple and inexpensive method that can be used to cryopreserve avian genetic material in the field. Future studies are required to determine whether progeny can be produced from vitrified GGCs.

\section{Acknowledgments}

We thank the staff at the Animal Science Division of the Agricultural and Forestry Research Center, University of Tsukuba, for technical assistance. This study was supported in part by a Grant-in-aid awarded to AT from the Ministry of Education, Culture, Sports, Science and Technology, Japan (16380184).

\section{References}

Bakst MR and Sexton TJ. Fertilizing capacity and ultrastructure of fowl and turkey spermatozoa before and after freezing. Journal of Reproduction and Fertility, 55: 1-7. 1979.

Chen SU, Lien YR, Cheng YY, Chen HF, Ho HN and Yang YS. Vitrification of mouse oocytes using closed pulled straws (CPS) achieves a high survival and preserves good patterns of meiotic spindles, compared with conventional straws, open pulled straws (OPS) and grids. Human Reproduction, 16: 2350-2356. 2001.

Eyal-Giladi H, Ginsburg M and Farbarov A. Avian primordial germ cells are of epiblastic origin. Journal of Embryology and Experimental Morphology, 65: 139-147. 1981.

Gee GF, Bakst MR and Sexton TJ. Cryogenic preservation of semen from the greater sandhill crane. Journal of Wildlife Management, 49: 480-484. 1985.

Hamburger V and Hamilton HL. A series of normal stage in the development of the chick embryo. Journal of Morphology, 8: 49-92. 1951.

Hodges J. Conservation of genes and culture: historical and contemporary issues. Poultry Science 85: 200-209. 2006.

IUCN Red List (2006) http://www.redlist.org/

Kasai M, Komi JH, Takakamo A, Tsunoda H, Sakurai T and Machida T. A simple method for mouse embryo cryopreservation in a low-toxicity vitrification solution, without 
appreciable loss of viability. Journal of Reproduction and Fertility, 89: 91-97. 1990.

Kong LK, Lee SI, Cho SG, Cho SK and Park CS. Comparison of open pulled straw (OPS) vs glass micropipette (GMP) vitrification in mouse blastocysts. Theriogenology, 53: 18171826. 2000.

Kuwana T. Migration of avian primordial germ cells toward the gonadal anlage. Development, Growth and Differentiation, 35: 237-243. 1993.

Kuwana T and Rogulska T. Migratory mechanisms of chick primordial germ cells toward gonadal anlage. Cellular and Molecular Biology, 45: 725-736. 1999.

Leibo SP and Oda K. High survival of mouse zygotes and embryos cooled rapidly or slowly in ethylene glycol plus polyvinylpyrrolidone. Cryo-Letters, 14: 133-144. 1993.

Maeda T, Terada T and Tsutsumi Y. Morphological observations on frozen and thawed muscovy spermatozoa. British Poultry Science, 25: 409-413. 1984.

Minematsu T, Tajima A and Kanai Y. The migratory ability of gonadal germ cells in the domestic chicken. Journal of Poultry Science, 41: 178-185. 2004.

Naito M, Tajima A, Tagami T, Yasuda Y and Kuwana T. Preservation of chick primordial germ cells in liquid nitrogen and subsequent production of viable offspring. Journal of Reproduction and Fertility, 102: 321-325. 1994.

Nakao K, Nakagata N and Katauki M. Simple and efficient vitrification procedure for cryopreservation of mouse embryos. Experimental Animals, 46: 231-234. 1997.

Polge C. Functional survival of fowl spermatozoa after freezing at $-79^{\circ} \mathrm{C}$. Nature, 167: 949-950. 1951.
Rall and Fahy. Ice-free cryopreservation of mouse embryos at $-196^{\circ} \mathrm{C}$ by vitrification. Nature, 313: 573-575. 1985.

Shaw PW, Bernard AG, Fuller BJ, Hunter JH and Shaw RW. Vitrification of mouse oocytes using short cryoprotectant exposure: effects of varying exposure time in survival. Molecular Reproduction and Development, 33: 210-214. 1992.

Swift $\mathrm{CH}$. Origin and early history of the primordial germ cells in the chick. American Journal of Anatomy, 135: 51-70. 1914.

Tachikawa S, Otoi T, Kondo S, Matida T and Kasai M. Successful vitrification of bovine blastocysts derived by in vitro maturation and fertilization. Molecular Reproduction and Development, 34: 266-271. 1993.

Tajima A, Naito M, Yasuda Y and Kuwana T. Production of germ line chimera by transfer of primordial germ cells in the domestic chicken (Gallus domesticus). Theriogenology, 40: 509-519. 1993.

Tajima A, Naito M, Yasuda Y and Kuwana T. Production of germ-line chimeras by transfer of cryopreserved gonadal primordial germ cells (gPGCs) in chicken. Journal of Experimental Zoology, 260: 265-267. 1998.

Ukeshima A and Fujimoto T. Ultrastructure of primordial germ cells in the early chick embryo. In: Ultrastructure of Reproduction: Gametogenesis, Fertilization, and Embryogenesis (Van Blerkom J and Motta PM Eds). pp. 12-18. Martinus Nijhoff Publishers, Boston. 1984.

Vajta G, Holm P, Kawayama M, Greve T and Callesen H. Open pulled straw (OPS) vitrification: a new way to reduce cryoinjuries of bovine ova and embryos. Molecular Reproduction and Development, 51: 53-58. 1998. 